\title{
Expanded endonasal approach: fully endoscopic, completely transnasal approach to the middle third of the clivus, petrous bone, middle cranial fossa, and infratemporal fossa
}

\author{
Amin B. Kassam, M.D., Paul Gardner, M.D., Carl Snyderman, M.D., \\ Arlan Mintz, M.Sc., M.D., AND Ricardo CaRraU, M.D. \\ Minimally Invasive Endoneurosurgical Center, University of Pittsburgh Medical Center, Pittsburgh, \\ Pennsylvania
}

\begin{abstract}
Object. The middle third of the clivus and the region around the petrous internal carotid artery (ICA) is a difficult area of the skull base in terms of access. This is a deep area rich with critical neurovascular structures, which is often host to typical skull base diseases. Expanded endoscopic endonasal approaches offer a potential option for accessing this difficult region. The objective of this paper was to establish the clinical feasibility of gaining access to the paraclival space in the region of the middle third of the clivus, to provide a practical modular and clinically applicable classification, and to describe the relevant critical surgical anatomy for each module.

Methods. The anatomical organization of the region around the petrous ICA, cavernous sinus, and middle clivus is presented, with approaches divided into zones. In an accompanying paper in this issue by Cavallo, et al., the anatomy of the pterygopalatine fossa is presented; this was observed through cadaveric dissection for which an expanded endonasal approach was used. In the current paper the authors translate the aforementioned anatomical study to provide a clinically applicable categorization of the endonasal approach to the region around the petrous ICA. A series of zones inferior and superior to the petrous ICA are described, with an illustrative case presented for each region.

Conclusions. The expanded endonasal approach is a feasible approach to the middle third of the clivus, petrous ICA, cavernous sinus, and medial infratemporal fossa in cases in which the lesion is located centrally, with neurovascular structures displaced laterally.
\end{abstract}

\section{KEY WORDS • endoscopy • endonasal approach • transsphenoidal approach • clivus • middle cranial fossa - petrous apex • meningioma}

The middle third of the clivus represents a challenging region in terms of access via conventional skull base approaches that follow a lateral-to-medial trajectory. Using the expanded endonasal approach, we have gained progressive access to this region from a medial-to-lateral direction via a fully endoscopic, completely transnasal route. The most critical anatomical landmark in this region is the course of the petrous and parasellar segments of the ICA. We have found it useful to classify the expanded endonasal approaches to this region based on their relationship to the petrous ICA. In this paper we provide a classification describing five modular approaches that provide access to several areas: the middle third of the clivus, petrous bone,

\footnotetext{
Abbreviations used in this paper: ICA = internal carotid artery; $\mathrm{LPP}=$ lateral pterygoid plate; MPP = medial pterygoid plate; V2 = second branch of the trigeminal nerve.
}

middle cranial fossa, inferior and superior portions of the cavernous sinus, and medial portion of the infratemporal fossa (Fig. 1).

The objectives of this paper are to establish the clinical feasibility of accessing the paraclival space in the region of the middle third of the clivus by using an expanded endonasal approach; to provide a practical modular and clinically applicable classification; and to describe the relevant critical surgical anatomy for each module. To further translate the anatomical understanding gained from the accompanying paper describing the cadaveric study, we also present an illustrative case example to demonstrate the technical nuances.

In general, it is useful to divide these five modular approaches into two general categories based on whether they are below the horizontal segment of the petrous ICA (infra- 


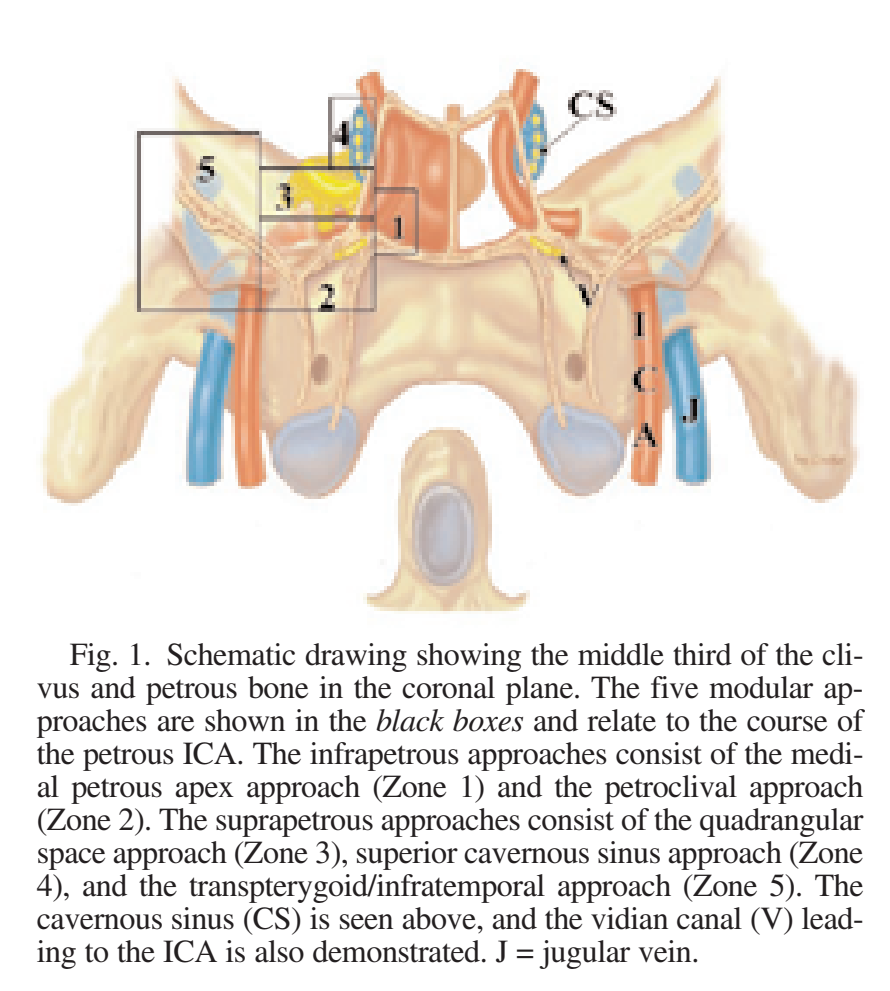

petrous approaches) or above it (suprapetrous approaches). The infrapetrous approaches consist of medial petrous apex and petroclival approaches (Zones 1 and 2, respectively, in Fig. 1). The suprapetrous approaches are the inferior cavernous sinus/middle cranial fossa approach (quadrangular space approach), superior cavernous sinus approach, and the transpterygoid approach to the infratemporal fossa. Zones 3, 4, and 5, respectively, in Fig. 1 represent these suprapetrous approaches.

\section{INFRAPETROUS APPROACHES}

\section{Zone 1: Medial Petrous Apex}

Access to the medial petrous apex with conventional approaches can be achieved via an extended middle cranial fossa approach or via transmastoid ones.,14,18,27,33-36 This can involve traction on the temporal lobe or having to dissect along the course of the facial nerve. Transnasal routes to the medial petrous apex have been reported ${ }^{13,25,28}$ and are gaining popularity with the advent of endoscopic approaches. These are particularly useful when the lesion has created a window by expanding the petrous apex medially toward the clivus. In these cases, the petrous apex remodeling protrudes into the sphenoidal sinus along the lateral sphenoid recess. This extension allows the lesion to intrude medially into the sphenoidal sinus beyond the genu of the ICA and provides direct access via the sphenoidal sinus.

Illustrative Case. In this 23-year-old man who presented with increasing headache, admission MR imaging revealed a cholesteatoma of the petrous bone (Fig. 2). The patient underwent an expanded endonasal medial petrous apex approach (Zone 1) for drainage. The procedure was uncom-

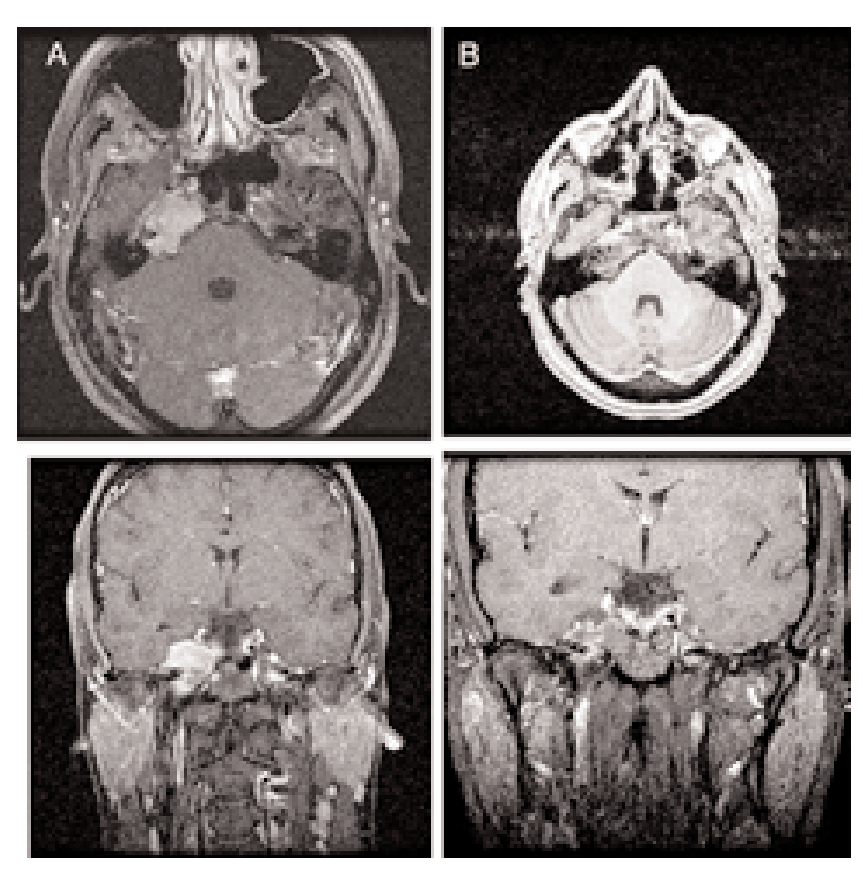

Fig. 2. Preoperative (A) and postoperative (B) MR images demonstrating a petrous cholesterol granuloma. A: Note that the lesion is hidden behind the anterior genu of the ICA, which guards access to it. The absence of the petrous apex remodeling requires mobilization of the ICA to gain access to the underlying petrous bone. The preoperative axial postcontrast MR image (upper) reveals the lesion under the ICA. The preoperative coronal postcontrast MR image (lower) reveals the lesion beneath the genu of the ICA in the infrapetrous position. B: The postoperative contrastenhanced axial (upper) and coronal (lower) MR images demonstrate drainage of the cholesterol granuloma.

plicated and he was discharged on postoperative Day 1. His headaches were subjectively improved at 1 week postoperatively, although long-term follow-up results are not yet available.

Technical Notes. If the petrous apex remodeling is not present as in the preceding illustrative case (Fig. 2), the exposure required for the expanded endonasal approach will mandate isolation of the anterior genu of the ICA and gentle lateral mobilization to access the underlying petrous bone. In these circumstances, the anterior genu of the ICA guards access to the petrous apex. The first step is to perform a wide bilateral endonasal approach to the sphenoid sinus as we have previously described. ${ }^{2,5}$ Once the bilateral sphenoidotomies are complete, the basopharyngeal fascia is stripped away from the undersurface of the sphenoid floor. The sphenoid floor is then drilled until it is flush with the clival recess. The sphenopalatine and posterior nasal arteries are isolated and ligated. A posterior maxillary antrostomy is performed and the MPP, which represents the key anatomical landmark for this module, is identified. The MPP is drilled in a straight line perpendicular to the clivus for approximately $1 \mathrm{~cm}$, and this leads directly to the anterior genu of the ICA as its petrous portion turns vertical (Fig. 3). The bone over the anterior genu of the ICA is then drilled to eggshell thinness with a coarse diamond 3-mm drill bit and the ICA is exposed. It is important to remove 
the bone lateral to the ICA to allow the vessel to be moved in a lateral direction without compressing and occluding it in the carotid canal. Once the ICA is moved laterally, the underlying petrous apex can be directly accessed. Drilling a portion of the clivus at its junction with the petrous apex can further facilitate this medial-to-lateral access (Video 1). This approach can provide access as far as the medial anterior margin of the internal auditory canal (Fig. 4).

Video 1: Zone 2, medial petrous apex approach. (Click here to view with Windows Media Player and a broadband connection, here to view with a dial-up connection, or here to view with RealPlayer.)

This approach is ideal for extradural lesions such as cholesterol granulomas and cholesteatomas. In these cases, marsupialization into the sphenoid sinus provides an ideal tract for drainage.

\section{Zone 2: Petroclival Approaches}

Conventional approaches to the petroclival region are well described and require a significant degree of petrous bone resection for access to these medial segments. ${ }^{1,9-12}$, 17,18,22,23 The expanded endonasal approach offers a direct route to lesions along the medial portion of the petroclival junction (these lesions can be either intradural or extradural). In this report, we restrict the technical description to the medial petroclival extradural region (Zone 2, Fig. 1).

Illustrative Case. This 60-year-old woman presented with progressive right abducent nerve palsy and headache. Admission MR imaging revealed an extradural lesion at the petroclival junction with a small intradural extension (Fig. 5A). Results of the metastatic workup were negative and the patient underwent an expanded endoscopic endonasal infrapetrous approach (Zone 2, Fig. 1). Significant venous bleeding was encountered from the basilar venous plexus and cavernous sinus during the resection, requiring blood transfusion (Video 2). Given her advanced age and the

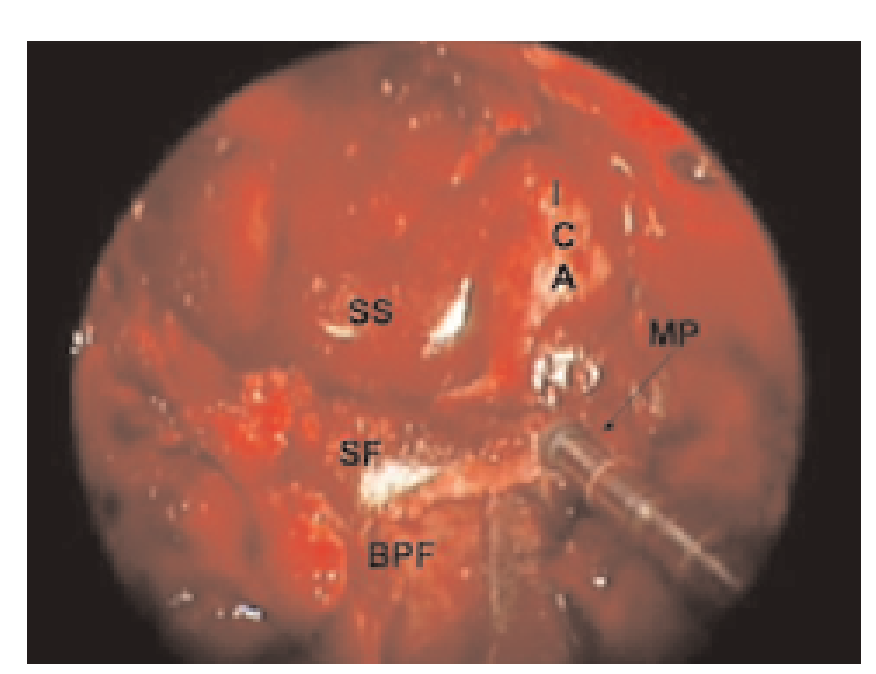

Fig. 3. Intraoperative endoscopic view showing isolation of the left MPP (MP) after the sphenoidal floor (SF) has been drilled flush with the clival recess. The MPP is drilled in a straight line until the ICA canal is encountered in the sphenoidal sinus (SS). This provides access to the anterior genu of the ICA. BPF = basopharyngeal fascia.

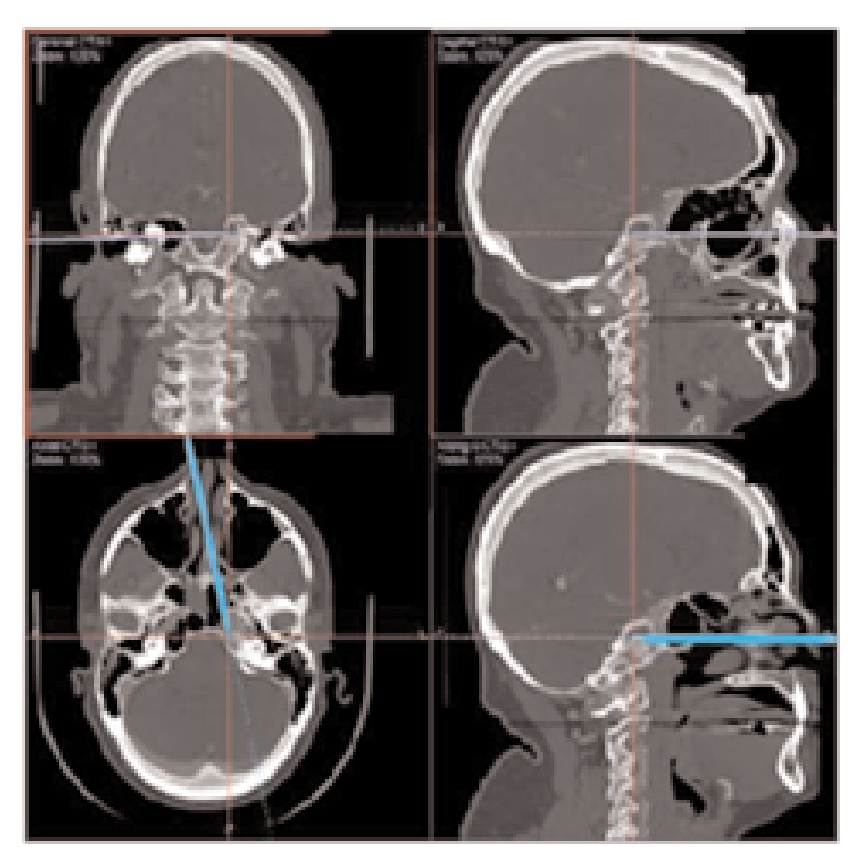

Fig. 4. Intraoperative image guidance is used to demonstrate the extent of the infrapetrous ICA access that can be achieved. Note the position of the probe just medial and superior to the margin of the internal auditory canal.
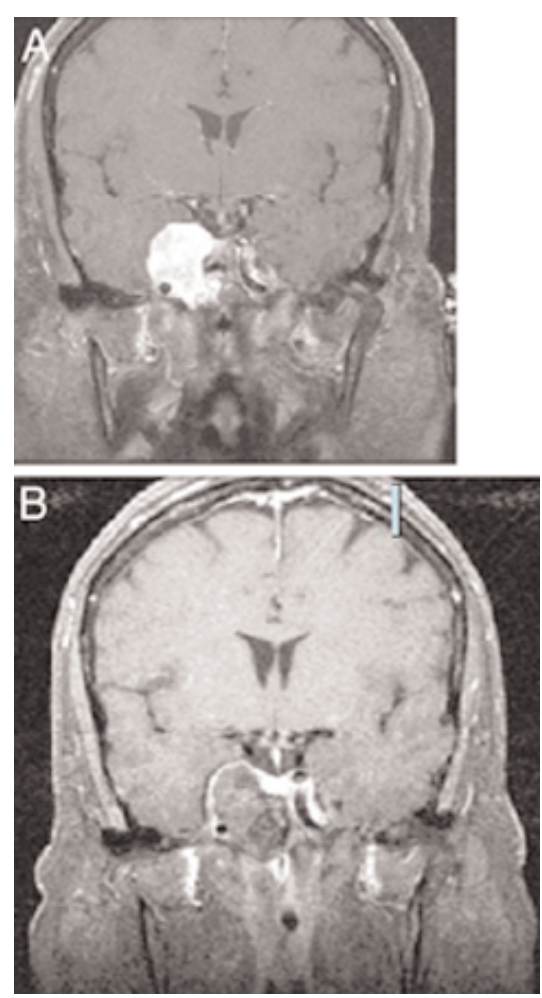

Fig. 5. Preoperative and postoperative contrast-enhanced MR images demonstrating a petroclival chondrosarcoma with extension into the inferior medial portion of the cavernous sinus. A: Coronal postcontrast MR image revealing the extent of the tumor. Note on the coronal image that the lesion extends more than $180^{\circ}$ around the medial border of the ICA. B: Postoperative coronal image demonstrating gross-total resection of the tumor. Note on the coronal view the complete removal of the tumor over the medial $180^{\circ}$ of the ICA as seen on the preoperative image. 
intraoperative blood loss, the patient was observed for 2 days postoperatively. The abducent nerve palsy remained stable and no new deficits occurred, although long-term follow-up results are not yet available.

Video 2: Zone 2, petroclival approach. (Click here to view with Windows Media Player and a broadband connection here to view with a dial-up connection, on here to view with RealPlayer.)

Technical Notes. The procedure is initiated as described in the previous petrous apex Zone 1 module. Once the sphenoid floor is drilled to the clival recess and the MPP is isolated, the petroclival module can begin. The basopharyngeal fascia is completely stripped away from the undersurface of the MPP as it transitions to the petrous bone laterally. To improve the access to these more caudally oriented regions, the posterior nasal septum is completely detached from the rostrum of the sphenoid (Fig. 6). This allows the septum to float freely, minimizing the contamination of the endoscope during bimanual dissection. The MPP is isolated and drilling is initiated along its inferior margin in a caudal-to-rostral direction (Fig. 7). It is imperative to perform the drilling along this caudal-to-rostral trajectory to avoid the horizontal segment of the ICA. As the drilling proceeds, the vidian canal is identified. A good landmark is the junction of the sphenoid floor with the MPP. Just lateral to this point, the vidian canal can be found (Fig. 7), and the vidian artery is also identified (Figs. 1 and 7). The drilling proceeds circumferentially along the vidian canal as the vidian artery is followed to the place where it emerges from the anterior genu of the ICA. This is the most important anatomical landmark for this module. The anterior genu can be isolated and the bone over the horizontal segment, genu, and parasellar carotid protuberance can be removed using the combination of a high-speed, extended drill and fine Kerrison rongeurs.

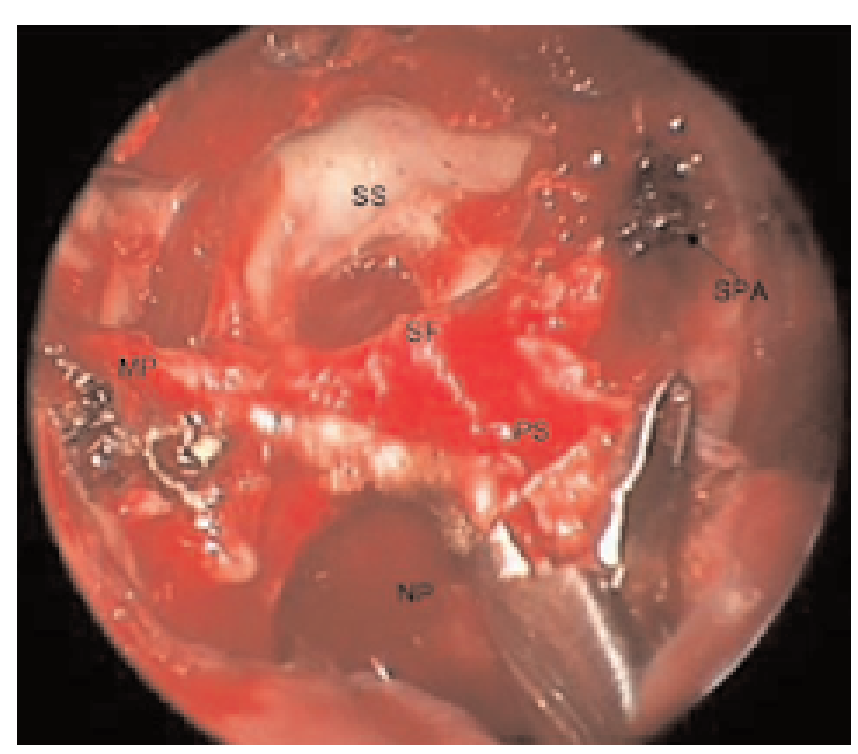

Fig. 6. Endonasal endoscopic view showing detachment of the posterior nasal septum (PS) from the rostrum. Note that the sphenopalatine artery (SPA) has been ligated and the MPP is isolated. Resection of the posterior nasal septum creates a single cavity rostrally from the sphenoidal sinus to the nasopharynx (NP) caudally.
The medial portion of the clivus at the petroclival junction is drilled until the underlying dura mater and venous plexus is identified (Fig. 8). This provides direct access to this region. Using the anterior genu of the ICA as the lateral margin, tumor removal should continue flush with the ICA. This portion of the tumor represents the segment along the medial $180^{\circ}$ of the ICA (Figs. 5 and 8 ). The resection can proceed along this segment and through the region of the Dorello canal, following the course of the abducent nerve as it runs obliquely to enter the cavernous sinus superolaterally. The cavernous sinus represents the superior boundary of this exposure and the middle fossa represents the lateral boundary. Venous bleeding from the cav-

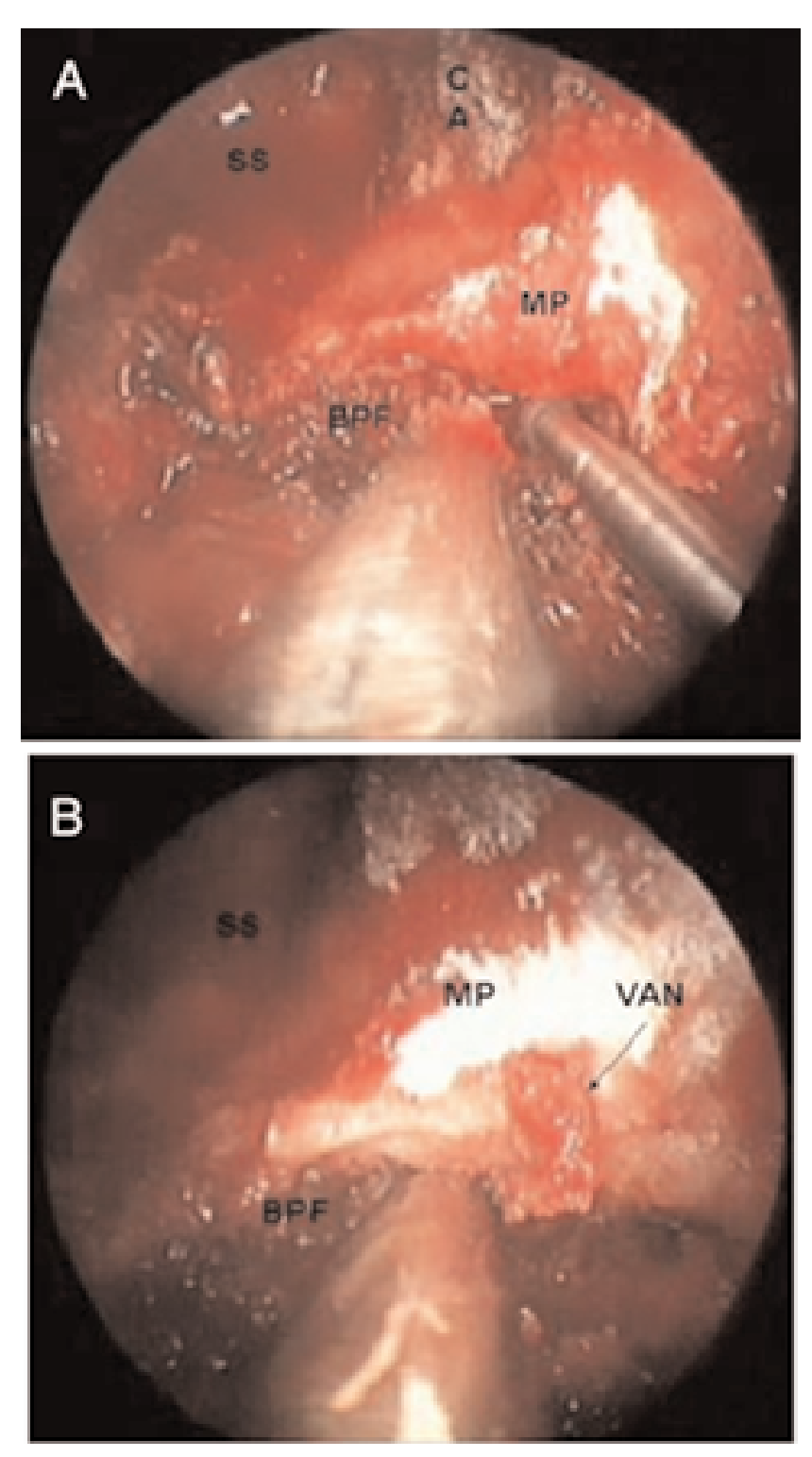

Fig. 7. A: Endonasal endoscopic view showing resection of the basopharyngeal fascia (BPF) and isolation of the left MPP. The MPP is drilled in a caudal-to-rostral direction toward the ICA (CA) at the level of the sphenoid sinus. B: Endonasal view showing isolation of the left vidian artery (VAN) in the vidian canal following drilling of the left MPP. 
ernous sinus and basilar venous plexus is controlled with Avitene packing. If required, the deep dura mater medial to the posterior margin of the cavernous sinus can be opened to provide access to the pontine cistern and the neurovascular structures within (Fig. 8, Video 2).

Reconstruction of this zone requires packing with Avitene for hemostasis, and placement of autograft composed of abdominal or thigh fat to protect the exposed ICA. If the dura mater is opened, an inlay and onlay graft is placed as we have previously described. ${ }^{21}$ We do use a temporary balloon stent reinforcement to prevent migration of the grafts as a part of this reconstruction. This device is usually a
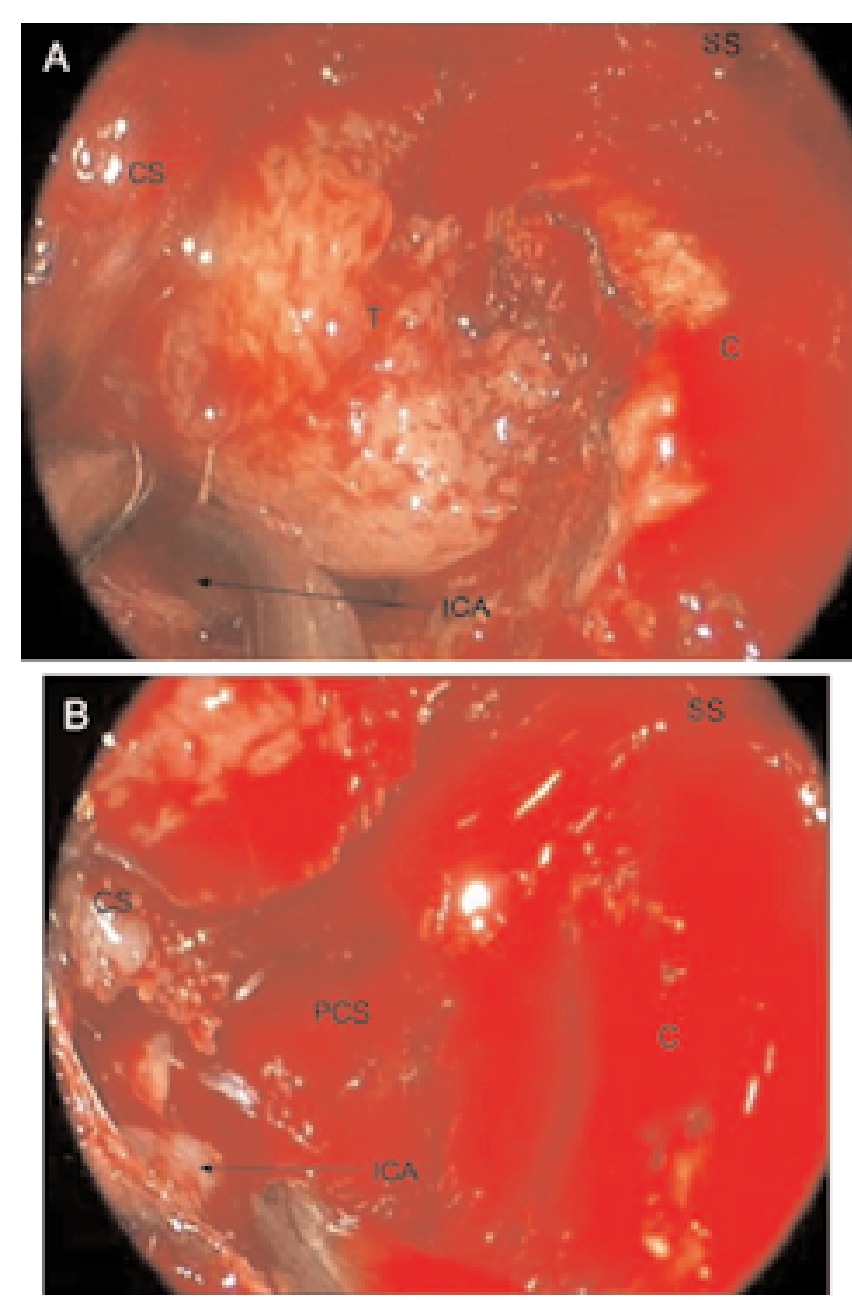

Fig. 8. A: Endonasal endoscopic view showing a right-sided Zone 2 approach. Note the firm fibrous tumor (T) at the right petroclival junction. The residual clivus $(\mathrm{C})$ can be seen medially. The portion of the clivus at the margin of the tumor cavity has been drilled. The sphenoidal sinus (SS) is seen above to the right and the inferior medial portion of the cavernous sinus (CS) with tumor involvement is seen to the 12-o'clock position. Note the ICA on the inferior lateral margin of the lesion. This represents the portion of the tumor that extends $180^{\circ}$ around the medial margin of the ICA. B: Endonasal endoscopic view obtained after removal of the portion of the tumor that extended $180^{\circ}$ around the medial margin of the ICA. The resection cavity in the posterior cavernous sinus (PCS) is seen. Residual tumor is seen within the inferior segment of the cavernous sinus that was subsequently resected. catheter balloon inflated carefully under endoscopic visualization.

We have found this approach most useful for chondrosarcomas, chordomas, and sinonasal lesions of the petroclival space. The expanded endonasal approach may prove to offer an advantage for these medially positioned lesions by providing a direct route to the tumor without the need for a lateral approach, minimizing the need for neurovascular manipulation. We have also used the approach for intradural, petroclival tumors such as meningiomas, but this is beyond the scope of this report.

\section{SUPRAPETROUS APPROACHES}

\section{Zone 3: Inferior Cavernous Sinus/Quadrangular Space Approach}

Access to the inferior portion of the cavernous sinus and the middle cranial fossa can be achieved via laterally oriented middle fossa and orbitocranial approaches. 12,17,24,26, 29-31,35 Based on a prior study ${ }^{7}$ and the expanded endonasal approach modules described earlier we have been able to gain access to this region effectively by extending the exposure described in the petroclival module in a rostral direction.

Illustrative Case. This 73-year-old man presented with severe headache and right abducent nerve palsy. The patient had undergone multiple transcranial and transsphenoidal resections for recurrent chordoma and had also received radiotherapy (Fig. 9). He underwent a suprapetrous (Zone 3) expanded endonasal approach (Fig. 1), followed by a radiosurgery boost for residual tumor. No new deficits were encountered and the patient was observed for 72 hours postoperatively for medical management of his disease, given his advanced age. The 6-month follow-up review has demonstrated good tumor control.

Technical Notes. The exposure is initiated as described in the petroclival (Zone 2) module. The $\mathrm{VC}$ is isolated and the maxillary antrostomy is widened laterally to expose the posterior wall of the maxilla and isolate the V2 (Fig. 10). The drilling is performed in the same direction as described earlier. The vidian artery is followed to the anterior genu of the ICA and then isolation of V2 is pursued. The V2 is then followed superiorly until the foramen rotundum is identified, and bone is removed until the V2 disappears into the dura mater of the middle cranial fossa. To accomplish this, the bone separating the V2 and vidian canal is drilled away toward the junction of the horizontal petrous segment of the ICA and its anterior genu. Therefore, the key anatomical landmark in this module is the V2. Care should be taken during bone removal, because the space between the V2 and the vidian canal diminishes in a conelike fashion down to a relatively narrow space separating the two in the depth of the cranial fossa.

The ICA is then followed laterally and inferiorly and the bone over its horizontal portion is removed. The ICA is followed superiorly from the genu and the bone over the parasellar carotid protuberance is removed. The lateral portion of the cavernous sinus can be found between this portion of the ICA and the point at which the V2 disappears into the dura mater (Fig. 11). The dura is opened in the space from a medial-to-lateral direction, that is, from the genu of the ICA toward the V2. The quadrangular space is 

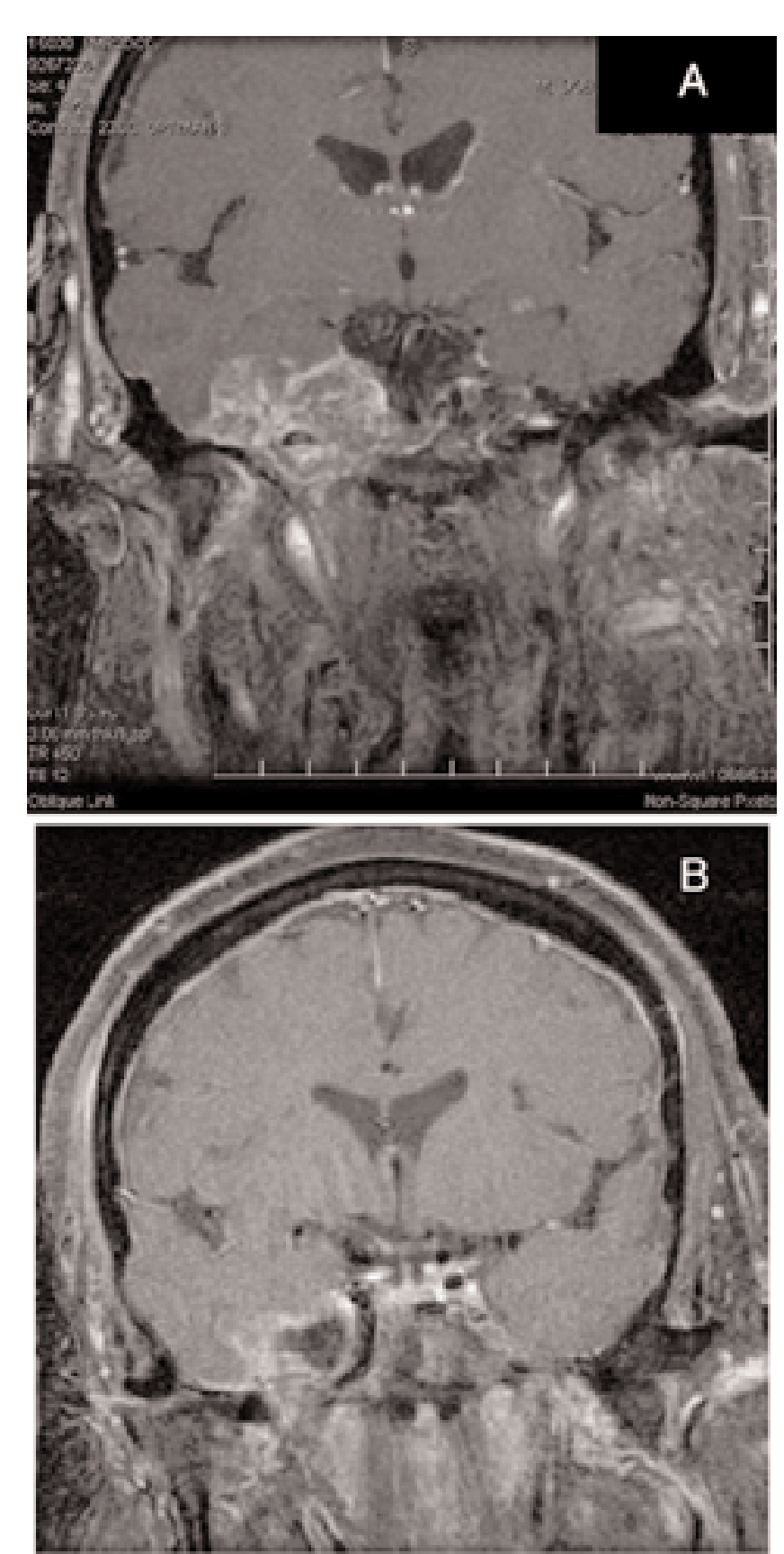

Fig. 9. A: Preoperative coronal postcontrast MR image demonstrating recurrent chordoma occupying the cavernous sinus with extension into the middle cranial fossa. B: Postoperative postcontrast coronal MR image demonstrating the resection cavity in the cavernous sinus and its extension into the middle fossa. Note that the medial margin of the resection extends to the lateral border of the cavernous segment of the ICA.

bounded by the ICA medially, by the V2 and dura mater of the middle fossa laterally, the horizontal petrous ICA inferiorly, and the course of the abducent nerve running obliquely and superiorly.

The dura mater in this space is opened widely and the tumor can be removed from this segment. Staying below the abducent nerve and lateral to the ICA allows one to
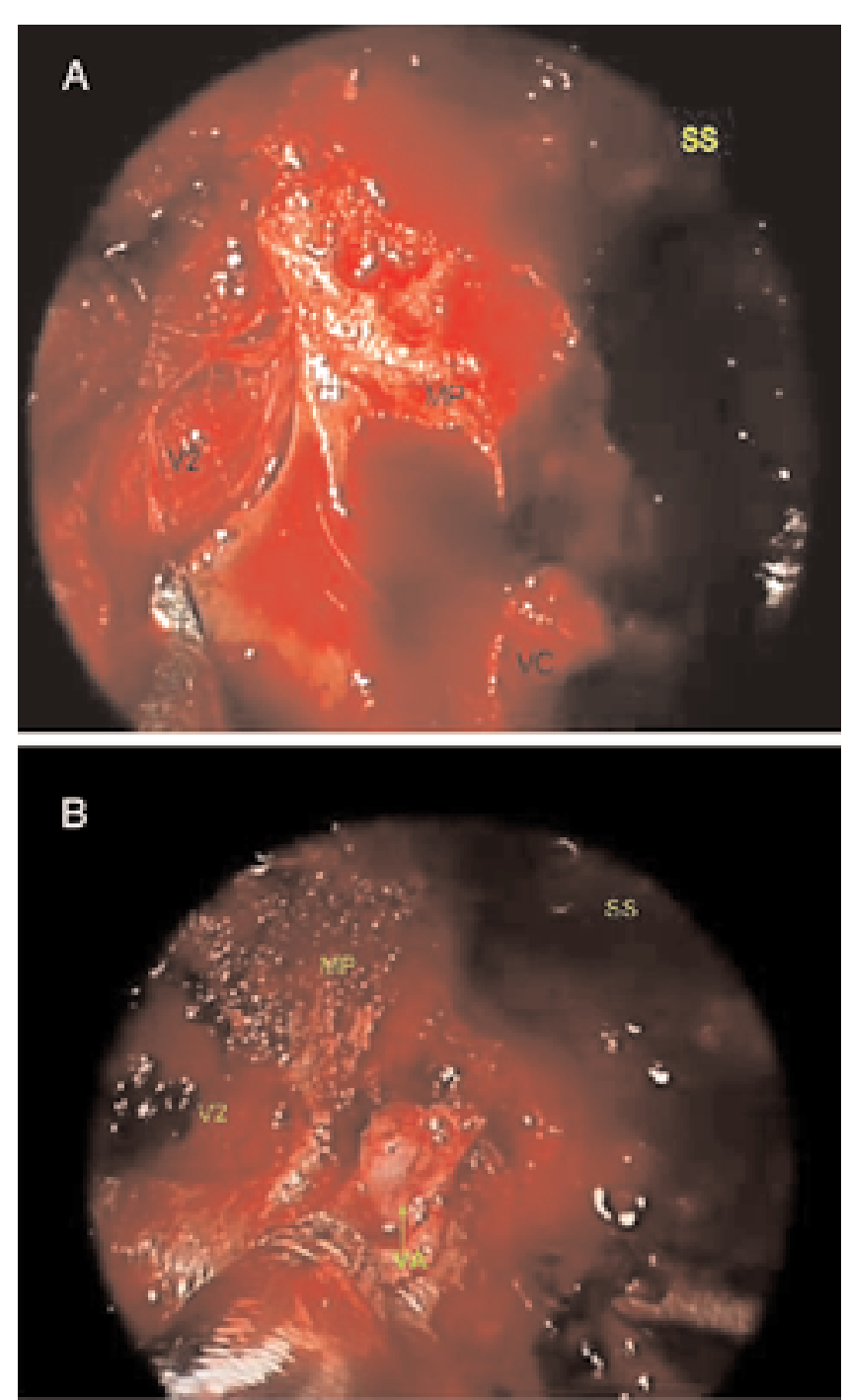

Fig. 10. A: Endonasal endoscopic view showing a right-sided Zone 3 approach. The right MPP has been isolated, and through the maxillary antrostomy the V2 can be seen. Inferiorly, the vidian canal (VC) has been isolated. Note the bone between V2 and the vidian canal that marks the region of the drilling, which leads to the quadrangular space. B: Endonasal endoscopic view showing isolation of the vidian artery (VA) and removal of the bone between it and the V2.

avoid the superior portion of the cavernous sinus, where the remainder of the cranial nerves are located. Tumor removal can continue in a posterior and lateral trajectory through the Meckel cave, the petrous bone, and into the posterior fossa as required. Angled endoscopes can be of value during this portion of the resection (Video 3 ).

Video 3: Zone 3, suprapetrous quadrangular approach.

(Click here to view with Windows Media Player and a broadband connection, here to view with a dial-up connection, or here to view with RealPlayer.)

Reconstruction of this segment is identical to that of the petroclival module. We have found this approach to be well 
suited for chordomas, chondrosarcomas, meningiomas, and sinonasal tumors. We believe this offers an advantage in situations in which the tumor is located medially and with neurovascular structures found along the lateral margins of the lesion. In these situations, the expanded endonasal approach provides direct access to this region. Venous bleeding from the cavernous sinus is controlled in the standard fashion with packing.

\section{Zone 4: Superior Portion of the Cavernous Sinus}
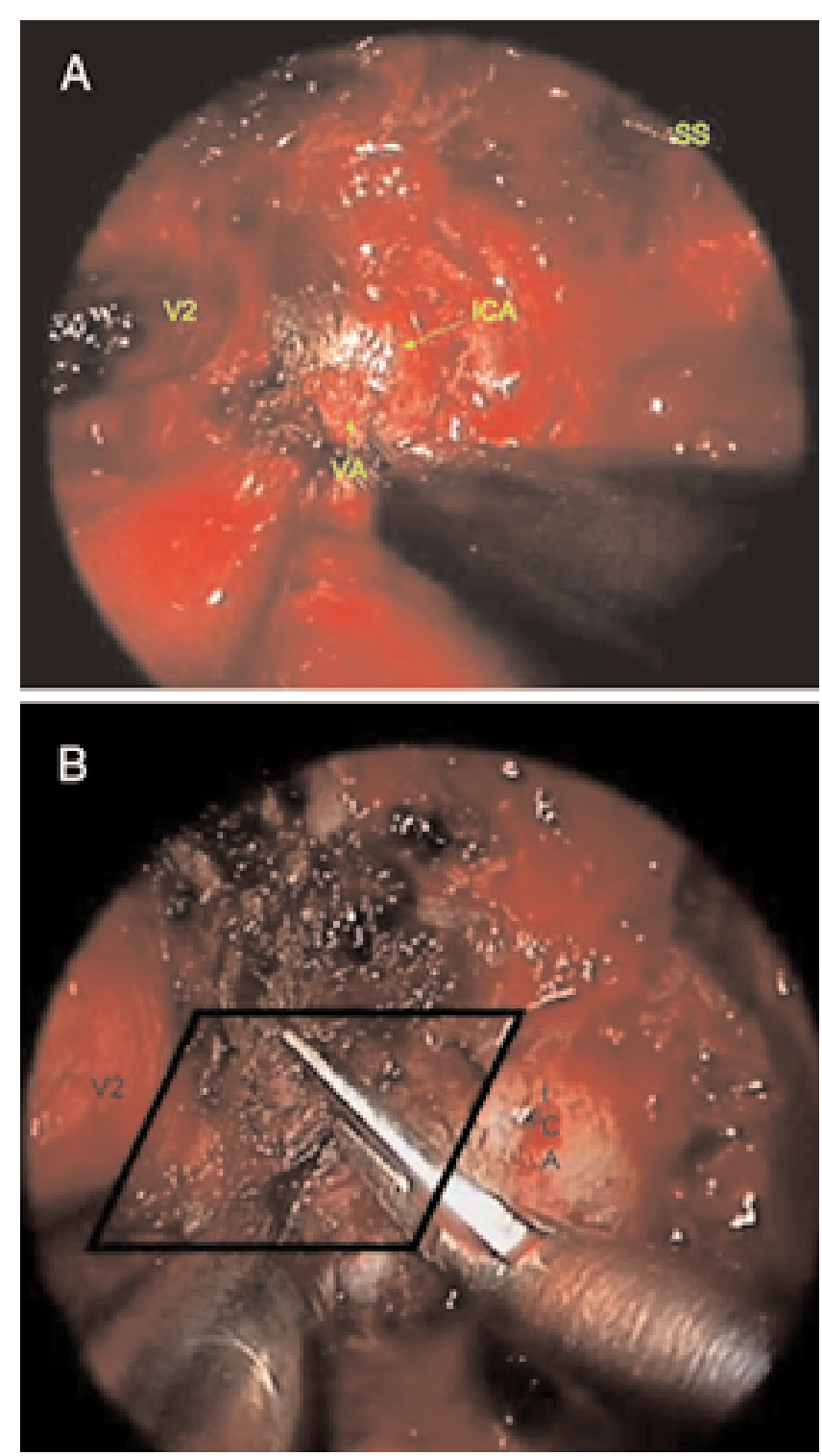

Fig. 11. A: Endonasal endoscopic view obtained after removal of the right MPP and bone between the V2 and the vidian artery (VA). The vidian artery is being cauterized and transected at its point of emergence from the anterior genu of the petrous ICA. B: Endonasal endoscopic view showing the right quadrangular space (black box) following resection of the stump of the vidian artery. The dural opening is made in a superolateral oblique direction away from the medially positioned ICA. The V2 and the dura mater of the middle cranial fossa represent the lateral extent of the opening.
Although the superior portion of the cavernous sinus does not directly relate to this segment of the clivus, the expanded endonasal approach used for access to this region represents a natural extension of the previous module.

Illustrative Case. This 47-year-old man presented with a
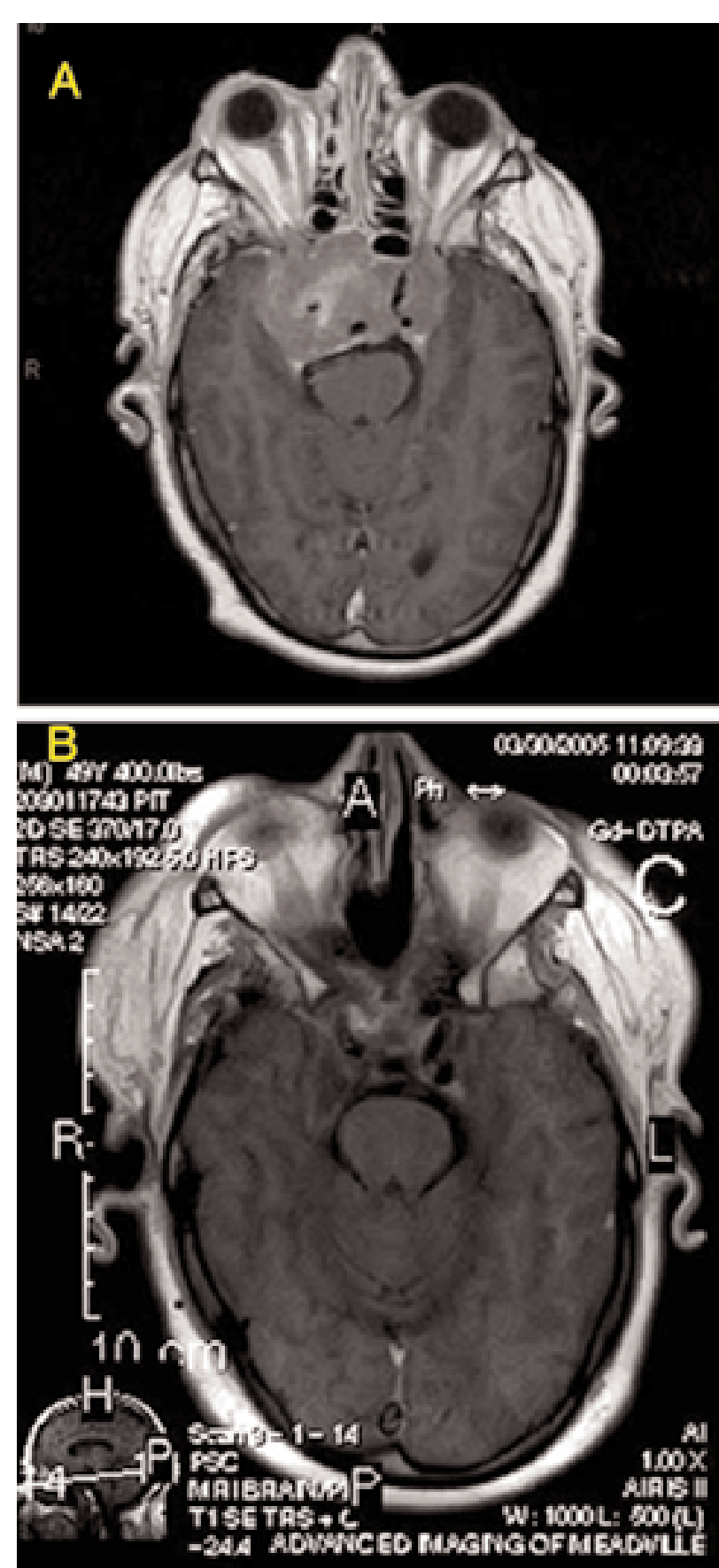

Fig. 12. A: Preoperative contrast-enhanced axial MR image demonstrating an invasive prolactinoma with cavernous sinus invasion. Note tumor between the right carotid artery loops. B: Two-year postoperative follow-up contrast-enhanced MR image. Note that the patient was maintained on medical therapy postoperatively. 
complete right ophthalmoplegia from a prolactin-secreting macroadenoma with cavernous invasion (Fig. 12). The patient had previously undergone transcranial surgery and his tumor was refractory to medical management. He underwent a suprapetrous (Zone 4) expanded endonasal approach (Fig. 1), from which no new deficits resulted. Postoperatively, the patient continued his medical regimen. At 6 months, he recovered his right oculomotor function. Two years later, he has a full range of extraocular movement in the affected eye and complete tumor control.

Technical Notes. The exposure of the ICA and bone removal is undertaken exactly as described in the quadrangular (Zone 3) module, with the only difference being the dural opening. For the Zone 4 module, the dural opening is made above the quadrangular space. In this case, an opening from a medial-to-lateral direction is initiated directly over the superior lateral portion of the cavernous sinus. In the majority of cases, the tumor has already thrombosed the sinus and little venous bleeding is encountered during the initial opening. It is often advisable to open the sella turcica and identify the medial margin of the ICA in the sella prior to opening the supralateral cavernous sinus. Once the ICA is identified medially, it can be protected with a dissector during the lateral opening.

Direct access to the cavernous sinus can be gained with this Zone 4 exposure. If needed, the quadrangular space (Zone 3) can also be opened to augment the exposure. Tumor can be stripped away from the cranial nerves (Fig. 13). Venous bleeding is encountered once the tumor is removed. The infralateral trunk of the ICA is a good landmark for the caudal extent of the exposure (Video 4).

Video 4: Zone 4, superior lateral cavernous sinus approach.

(Click here to view with Windows Media Player and a broad-

band connection here to view with a dial-up connection, or

here to view with RealPlayer.)

We have reserved this module for those rare tumors that are refractory to medical or radiosurgical management, or for patients with preexisting cranial nerve deficits. This has been useful for a very select subset of invasive pituitary macroadenomas and refractory meningiomas. We do not advocate the routine excision of tumors of the cavernous sinus.

\section{Zone 5: Transpterygoid/Infratemporal Approach}

Lateral approaches to the infratemporal fossa are well described and useful for extracranial lesions even if they have intracranial extension., $4,6,15,16,19,20,32,36$ They do require soft-tissue dissection and orbitocranial bone removal that, in general, are well tolerated. Issues related to mastication, aesthetics, and hypesthesia along the trigeminal nerve distribution are occasional consequences of this approach. Tumors with significant intracranial extension will require some degree of temporal lobe manipulation. As an alternative, in carefully selected cases in which the lesion is medial and inferior to critical neurovascular structures, the expanded endonasal approach offers a direct route, avoiding some of these issues.

Illustrative Cases. This 34-year-old woman presented with a left abducent nerve palsy and hypesthesia in the trigeminal (V2 and V3) distributions. Admission MR imaging revealed an 8-cm lesion extending from the left sylvian fis-

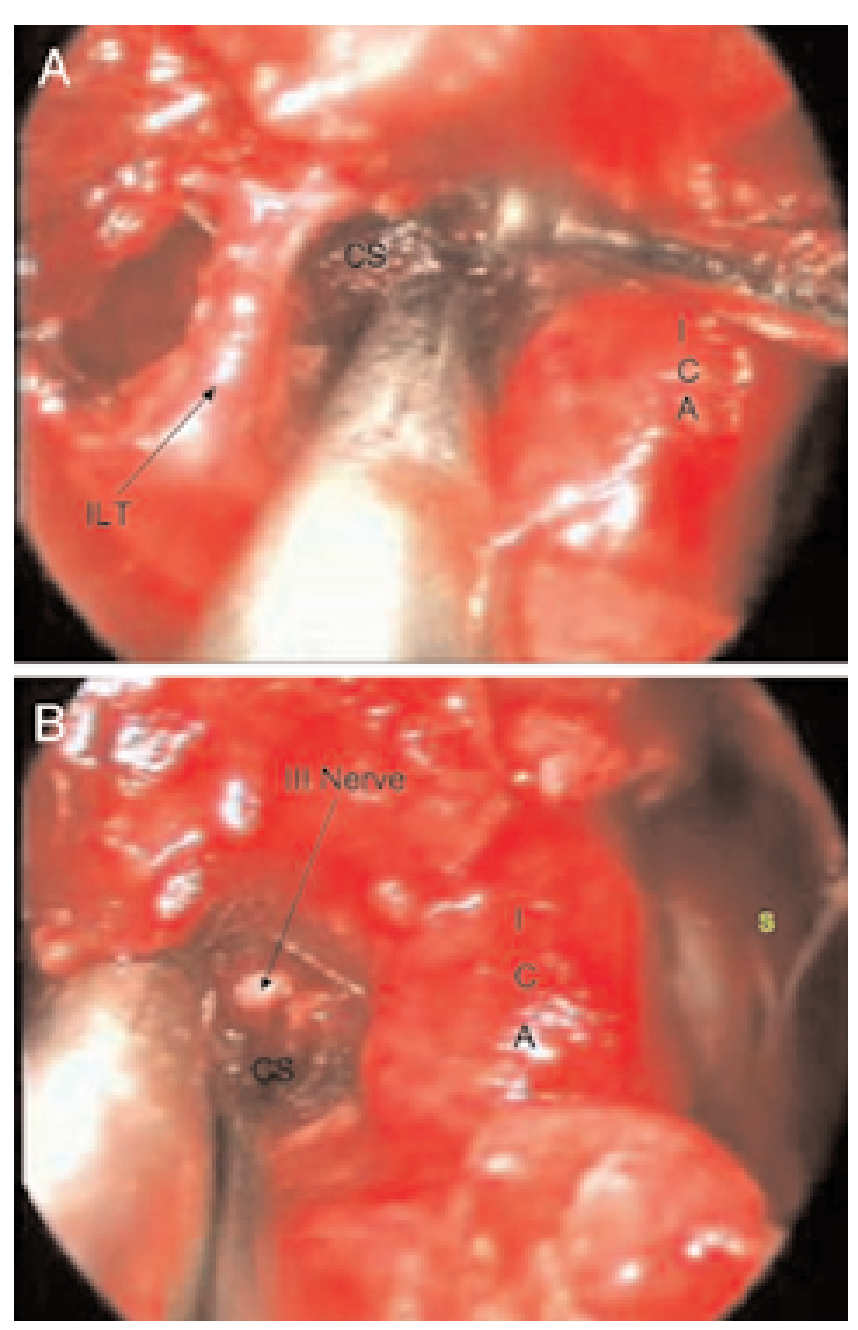

Fig. 13. A: Endonasal endoscopic view showing a right superior cavernous sinus dissection. The inferior lateral trunk (ILT) can be seen emerging from the ICA. Tumor in the cavernous sinus is being removed along the third cranial nerve between the inferior lateral trunk and the ICA. B: Endonasal view with the endoscope positioned between the inferior lateral trunk laterally and the ICA medially, showing tumor removal over the oculomotor nerve (III nerve). $\mathrm{S}=$ sellar.

sure through the infratemporal fossa. There was upward elevation of the left middle cerebral artery (Fig. 14). The patient underwent a three-stage, fully transnasal, completely endoscopic suprapetrous (Zone 5) approach. No new deficits were encountered. We elected to leave a small residual tumor in the superior portion of the cavernous sinus (Zone 4) to minimize the risk of oculomotor and trochlear nerve palsy. This portion was subsequently treated with radiosurgery. The 1-year follow-up review has shown no evidence of tumor growth.

Technical Notes. This module begins once the MPP is isolated and the maxillary antrostomy is completed. The tumor will often create the corridor through the maxillary sinus, extending rostrally to the middle cranial fossa and laterally to the infratemporal fossa. The MPP is identified and resected until it is flush with the middle cranial fossa 

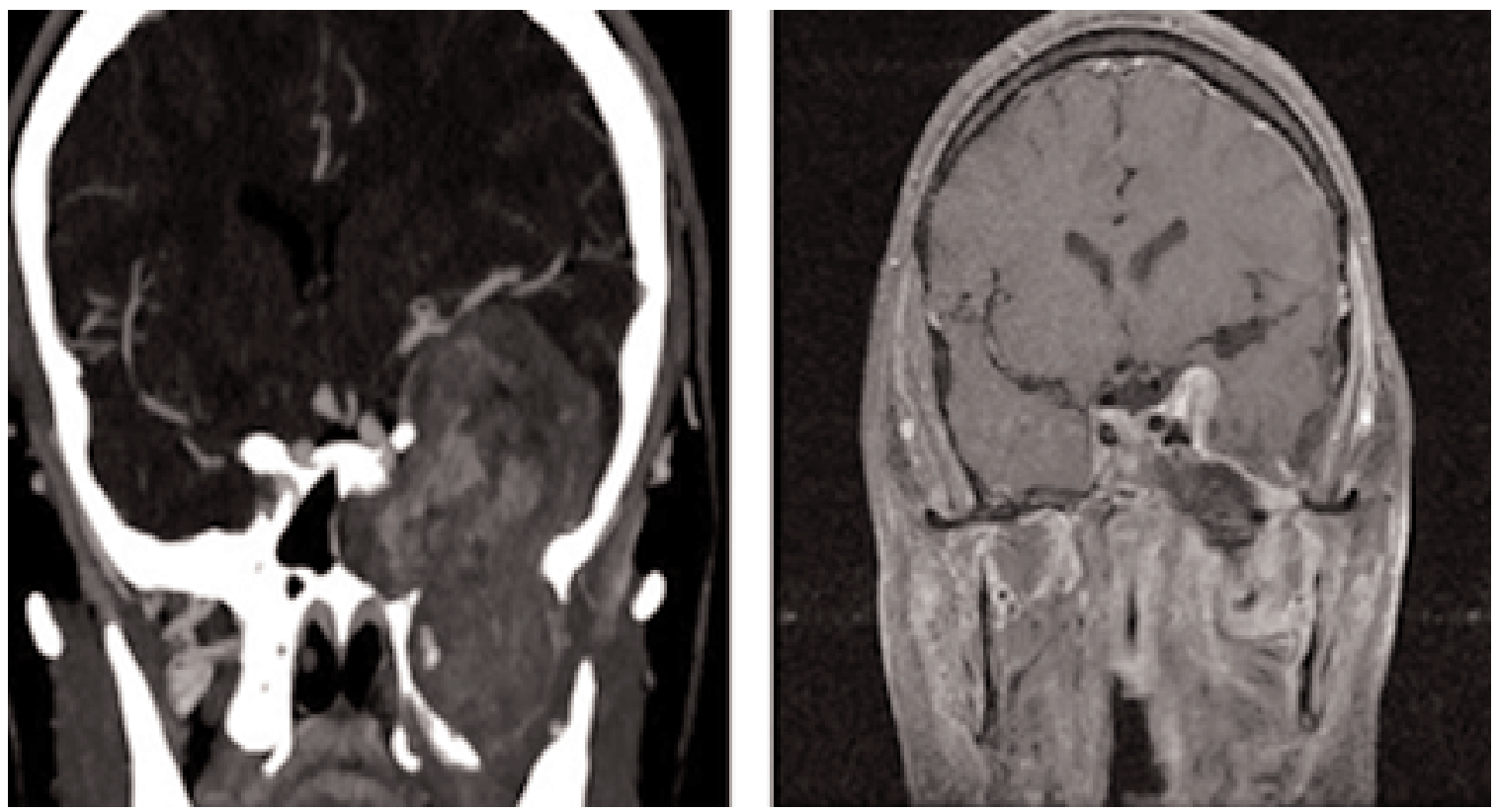

Fig. 14. Preoperative computerized tomography angiography (left) demonstrating a large schwannoma extending from the sylvian fissure through the infratemporal fossa. Note the significant elevation of the left middle cerebral artery. The postoperative contrast MR image (right) demonstrates a small amount of residual tumor in the superior cavernous sinus that we elected to leave.

and foramen rotundum as described previously. The tumor is encountered and once the anterior genu of the ICA and the horizontal/petrous segments of the ICA are identified, internal debulking of the lesion is initiated. During the lateral extracapsular dissection, the internal maxillary artery and its branches must be systematically isolated and ligated. The dissection is pursued laterally until the LPP is identified. The LPP is drilled rostrally until it is flush with the middle cranial fossa and foramen ovale.

Dissection in this space requires the use of a series of angled endoscopes, with much of the work being done with the $45^{\circ}$ and $70^{\circ}$ angled devices. It is common to encounter profuse venous bleeding from the pterygopalatine venous plexus, and this is controlled with packing. On occasion one may have to pack this plexus and return 48 hours later to allow thrombosis to occur. The anatomy in this space can be difficult to navigate because the osseous landmarks (MPP and LPP) are often destroyed by the tumor. Imaging guidance is imperative for this module.

We have found this exposure to be very useful for large trigeminal schwannomas that extend into the infratemporal fossa. If there is cavernous sinus extension that warrants resection, then one of the previously described modules can be added. This is also a useful exposure for encephaloceles and cerebrospinal fluid leaks of the middle cranial fossa.

\section{DISCUSSION}

The expanded endonasal approach represents an additional tool that is of value when medial-to-lateral approaches are required to gain access to lesions in which the critical neurovascular structures are located along the perimeter. This trajectory has the potential of providing a direct route into the lesion, minimizing the need to manipulate struc- tures located circumferentially along the perimeter. The primary contraindication to the approach is encountered when the critical neurovascular structures are located medial or ventral to the tumor, requiring manipulation before entering the lesion. For example, we believe this is why schwannomas represent a contraindication to the expanded endonasal approach, because the facial nerve will be directly in front of the tumor when a ventral approach is used. We have not found tumor size, vascularity, fibrosity, or calcification to represent contraindications. Using the expanded endonasal approach we have been able resect large tumors as well as significantly calcified lesions, such as chondrosarcomas. In this report we have restricted the discussion to the technical considerations associated with approaches.

Despite all else, an important contraindication to the expanded endonasal approaches is the surgeon's experience with the approach. The learning curve, particularly in this region, is extremely steep and requires team surgery that includes an otolaryngologist experienced in endoscopy and a neurosurgeon experienced in endoscopy, cranial base, and cerebrovascular surgery. The modules we have described build on each other with progressive dissection along the coronal plane requiring an understanding of the petrous segment of the ICA as visualized from this ventral vantage point. According to our new classification system all of these zones (with the exception of Zone 1)represent technically more advanced procedures (Kassam, et al., unpublished data). We believe that these higher-level procedures should be pursued only after the surgical team has developed an incremental experience with the previous ones. Once this anatomy is mastered, we believe the medial third of the clivus and the coronal extensions described in this paper are ideally suited for expanded endonasal approaches. This is particularly true if the lesion is extradural. 


\section{CONCLUSIONS}

In this paper we detail an anatomical classification that provides a practical guide for access to the paraclival regions surrounding the middle third of the clivus via a fully endoscopic, completely transnasal approach. The key anatomical landmarks are provided for each of a series of five modular approaches related to the petrous segment of the ICA. Thus, this paper provides a translation of the anatomical principles from the previous cadaveric study of Cavallo, et al. ${ }^{8}$ to a clinical setting. These modules can be used in isolation or in combination, based on the extent of the lesion.

Having established the clinical feasibility of access to this region by using the expanded endonasal approach, we have collected clinical outcome data in 23 patients, which will be reported separately (Kassam, et al., forthcoming). The experience is limited and a relatively small number of cases are included in each module. We are currently collecting long-term follow-up data in these cases and prospective data in additional cases to determine outcomes such as surgical cure, tumor control, and complications.

\section{References}

1. Abdel Aziz KM, Sanan A, van Loveren HR, et al: Petroclival meningiomas: predictive parameters for transpetrosal approaches. Neurosurgery 47:139-152, 2000

2. Al-Nashar IS, Carrau RL, Herrera A, et al: Endoscopic transnasal transpterygopalatine fossa approach to the lateral recess of the sphenoid sinus. Laryngoscope 114:528-532, 2004

3. Brackmann DE, Toh EH: Surgical management of petrous apex cholesterol granulomas. Otol Neurotol 23:529-533, 2002

4. Browne JD, Jacob SL: Temporal approach for resection of juvenile nasopharyngeal angiofibromas. Laryngoscope 110: 1287-1293, 2000

5. Carrau RL, Kassam AB, Snyderman CH: Pituitary Surgery. Otolaryngol Clin North Am 34:1143-1155, 2001

6. Cass SP, Hirsch BE, Stechison MT: Evolution and advances of the lateral surgical approaches to cranial base neoplasms. J Neurooncol 20:337-361, 1994

7. Cavallo LM, Cappabianca P, Galzio R, et al: endoscopic transnasal approach to the cavernous sinus versus transcranial route: anatomic study. Neurosurgery 56 (Suppl 2):379-389, 2005

8. Cavallo LM, Messina A, Gardner P, et al: Extended endoscopic endonasal approach to the pterygopalatine fossa: anatomical study and clinical considerations. Neurosurg Focus 19(1):E5, 2005

9. Chanda A, Nanda A: Partial labyrinthectomy petrous apicectomy approach to the petroclival region: an anatomic and technical study. Neurosurgery 51:147-160, 2002

10. Cho CW, Al-Mefty O: Combined petrosal approach to petroclival meningiomas. Neurosurgery. 51:708-718, 2002

11. Day JD, Fukushima T, Giannotta SL: Innovations in surgical approach: lateral cranial base approaches. Clin Neurosurg 43: 72-90, 1996

12. DiNardo LJ, Pippin GW, Sismanis A. Image-guided endoscopic transsphenoidal drainage of select petrous apex cholesterol granulomas. Otol Neurotol 24:939-941, 2003

13. Eisenberg MB, Haddad G, Al-Mefty O: Petrous apex cholesterol granulomas: evolution and management. J Neurosurg 86: 822-829, 1997

14. Fisch U: Infratemporal fossa approach to tumors of the temporal bone and base of the skull. J Laryngol Otol 92:949-967, 1978

15. Fisch U, Fagan P, Valavanis A: The infratemporal fossa approach for the lateral skull base. Otolaryngol Clin North Am 17:513-552, 1984
16. Fukushima T, Day JD, Hirahara K: Extradural total petrous apex resection with trigeminal translocation for improved exposure of the posterior cavernous sinus and petroclival region. Skull Base Surg 6:95-103, 1996

17. Gianoli GJ, Amedee RG. Hearing results in surgery for primary petrous apex lesions. Otolaryngol Head Neck Surg 111: 250-257, 1994

18. Goel A, Muzumdar D, Raman C: Trigeminal neuroma: analysis of surgical experience with 73 cases. Neurosurgery 52: 783-790, 2003

19. Hadley KS, Shelton C: Infratemporal fossa approach to the hypoglossal canal: practical landmarks for elusive anatomy. Laryngoscope 114:1648-1651, 2004

20. Hegazy HM, Carrau RL, Snyderman CH, et al: Transnasal endoscopic repair of cerebrospinal fluid rhinorrhea: a meta-analysis. Laryngoscope 110:1166-1172, 2000

21. Horgan MA, Anderson GJ, Kellogg JX, et al: Classification and quantification of the petrosal approach to the petroclival region. J Neurosurg 93:108-112, 2000

22. Horgan MA, Delashaw JB, Schwartz MS, et al: Transcrusal approach to the petroclival region with hearing preservation. Technical note and illustrative cases. J Neurosurg 94: 660-666, 2001

23. Inoue T, Rhoton AL Jr, Theele D, et al: Surgical approaches to the cavernous sinus: a microsurgical study. Neurosurgery 26: 903-932, 1990

24. Kacker A, Komisar A, Huo J, et al: Transsphenoidal surgery utilizing computer-assisted stereotactic guidance. Rhinology 39: 207-210, 2001

25. Pontius AT, Ducic Y: Extended orbitozygomatic approach to the skull base to improve access to the cavernous sinus and optic chiasm. Otolaryngol Head Neck Surg 130:519-525, 2004

26. Santoro A, Salvati M, Vangelista T, et al: Fronto-temporoorbito-zygomatic approach and variants. Surgical technique and indications. J Neurosurg Sci 47:141-147, 2003

27. Schulz T, Schneider JP, Bootz F, et al: Transnasal and transsphenoidal MRI-guided biopsies of petroclival tumors. J Magn Reson Imaging 13:3-11, 2001

28. Sekhar LN, Burgess J, Akin O: Anatomical study of the cavernous sinus emphasizing operative approaches and related vascular and neural reconstruction. Neurosurgery 21:806-816, 1987

29. Sekhar LN, Moller AR: Operative management of tumors involving the cavernous sinus. J Neurosurg 64:879-889, 1986

30. Sekhar LN, Schramm VL Jr, Jones NF: Subtemporal-preauricular infratemporal fossa approach to large lateral and posterior cranial base neoplasms. J Neurosurg 67:488-499, 1987

31. Sen CN: Tumors of the cranial base, in Rengachary SS, Wilkins RH (eds): Principles of Neurosurgery. London: Wolfe, 1994

32. Sennaroglu L, Slattery WH III: Petrous anatomy for middle fossa approach. Laryngoscope 113:332-342, 2003

33. Steward DL, Choo DI, Pensak ML: Selective indications for the management of extensive anterior epitympanic cholesteatoma via combined transmastoid/middle fossa approach. Laryngoscope 110:1660-1666, 2000

34. Terao T, Onoue H, Hashimoto T, et al: Cholesterol granuloma in the petrous apex: case report and review. Acta Neurochir 143:947-952, 2001

35. Yasuda A, Campero A, Martins C, et al: microsurgical anatomy and approaches to the cavernous sinus. Neurosurgery 56 (Suppl 1):4-27, 2005

36. Zhang M, Garvis W, Linder T, et al: Update on the infratemporal fossa approaches to nasopharyngeal angiofibroma. Laryngoscope 108: 1717-1723, 1998

Manuscript received May 31, 2005.

Accepted in final form June 28, 2005.

Address reprint requests to: Amin B. Kassam, M.D., University of Pittsburgh School of Medicine, 203 Lothrop Street, Suite 500, Pittsburgh, Pennsylvania 15213. email: kassamab@upmc.edu. 